\title{
Investigation on Using Low Voltage Automatic Regulation to Minimize the Impacts of Charging Plug-in Electric Vehicles in Distribution Systems
}

\author{
Priscila Costa Nascimento $^{1}$, Michel Girotto de Oliveira ${ }^{1}$ and José Carlos M. Vieira ${ }^{1}$ \\ ${ }^{1}$ Department of Electrical and Computer Engineering \\ São Carlos School of Engineering (EESC) \\ University of São Paulo (USP), 13566-590 São Carlos (Brazil) \\ Phone/Fax number:+55 16 3373-9997, e-mail: priscilacosta@usp.br, michelgirotto@usp.br, jcarlos@sc.usp.br
}

\begin{abstract}
The growth of micro and mini distributed generation and, more recently, the use of electric energy storage systems and the incentives for electric mobility are important examples of the transformations that distribution networks have been going through. In this context, this paper firstly presents the impacts of uncoordinated plug-in electric vehicles (PEVs) charging in a real Brazilian distribution system. Four scenarios were elaborated with different PEVs penetration levels and the results show increased voltage unbalance, system losses, and violations of the steady-state voltage limits, even in the presence of an automatic voltage regulator installed in the medium voltage network. Then, as the main contribution, the potential usage of automatic voltage regulation at the low voltage network was investigated to minimize the negative impacts of uncontrolled PEV charging on distribution system steady-state operation. It is important to highlight that this is not a common practice of utilities in Brazil. The obtained results showed that regulating the voltage at the low voltage side could be an effective solution to keep the voltages within statutory limits.
\end{abstract}

Keywords. Electric vehicles, electric power distribution system, low voltage regulation, voltage unbalance, power losses.

\section{Introduction}

In recent decades, measures have been adopted worldwide to reduce greenhouse gas emissions. In this context, due to decarbonization policies, electric vehicles (EVs) are proliferating globally at an accelerated pace, as they are a more sustainable automotive alternative than traditional ones [1]. These can be classified into three distinct groups, according to the form of energy supply used by them. The first put together the pure electric vehicles or the battery EVs (BEVs), the second group comprises the hybrid electric vehicles (HEVs), and the third contains the plug-in hybrid electric vehicles (PHEVs). The first and last ones are the study object of this paper and will be called only plug-in electric vehicles (PEVs).

One of the benefits of EVs is the possibility of diversifying the use of fuels concerning urban mobility, reducing dependence on fossil fuels [2]. Therefore, the main motivations for promoting EVs expansion are focused on environmental issues. However, charging PEVs can cause negative impacts on the power grid. Studies about the impacts resulting from the PEVs charging in distribution systems (DSs) were previously performed [3]-[5]. The results showed negative impacts on the DS for different PEVs penetration levels $\left(P_{l}\right)$. However, the impacts on the DS voltage regulation were not considered, because the regulating devices were not modeled. In [4], a model of PEV charging and residential power demand is developed. The PEV charging model has a 10-min resolution and is developed for two levels of residential charging. This model was used in this work to create different charging profiles of the PEVs.

In this context, this paper aims at identifying the impacts of uncontrolled charging PEVs on the voltage regulation, grid losses and voltage unbalances of a real Brazilian distribution system, equipped with an automatic voltage regulator at the medium voltage (MV) side. The results showed that PEVs can cause severe negative impacts, especially on the voltage steady-state limits, even in the presence of MV automatic voltage regulation. Then, as the main contribution, we investigated the potential of using automatic voltage regulation at the low voltage (LV) side (low voltage regulation - LVR) to keep the nodal voltages within statutory limits. It is important to highlight that controlled PEV charging strategies may not be available to PEVs owners, thus applying grid-based solutions, such as LVR, can be an interesting and feasible approach. Although using LVR is not a common practice of utilities in Brazil, some utilities already use them as an alternative for customers' compensation, reducing company costs and improving the service quality. Furthermore, low voltage regulation has been addressed in some works to provide a more effective voltage control in low voltage networks, as seen in [6], [7].

This paper is organized as follows. Section II provides the main characteristics of the studied system and the PEV model used. Section III describes the methodology to define and run the simulations. The results achieved 
before and after the implementation of the LVR devices are presented and analyzed in Section IV. Finally, Section $\mathrm{V}$ concludes the paper.

\section{Distribution Systems and PEV Model}

The electrical system used to study the impacts of PEVs was a real Brazilian radial DS, with an MV system and 47 LV networks (i.e., systems with voltages below $1.0 \mathrm{kV}$ ), modeled by [8] in the OpenDSS software [9]. Table I shows the key characteristics of these systems.

Table I. - Characteristics of the test system

\begin{tabular}{|l|c|}
\hline \multicolumn{1}{|c|}{ Name } & Data \\
\hline Length & $4.71 \mathrm{~km}$ \\
\hline Short-circuit level at the substation transformer & $525 \mathrm{MVA}$ \\
\hline Voltage at the MV level $^{\text {Number of MV customers }}$ & $13.8 \mathrm{kV}$ \\
\hline Numb & 159 \\
\hline Voltage at the LV level $^{1}$ & $0.22 \mathrm{kV}$ \\
\hline Peak load of LV customers & $4.1 \mathrm{MW}$ \\
\hline Number of LV customers & 1,659 \\
\hline
\end{tabular}

${ }^{1}$ The peak load refers exclusively to LV customers because the PEVs are allocated at the LV networks.

The system under study did not present any automatic voltage regulator in its original version provided by [8]. However, in this paper, an automatic voltage regulator was inserted into the MV network, located on the middle of the feeder's main trunk. The computational model used to model the regulator was available in the OpenDSS software, and it consists of a regulator for each phase of the system, with a regulation range of $\pm 10 \%$, in 32 steps (nominal voltage is $13.8 \mathrm{kV}$ ). Each wye-connected voltage regulator was sized and connected to $2000 \mathrm{kVA}$ transformers (designed to maintain an output voltage at $1.03 \mathrm{pu}$ - this value was chosen based on the maximum voltage limit determined by the regulatory agency of 1.05 $\mathrm{pu}$ and set slightly lower to not cause voltage violations in unbalanced system loads). Furthermore, the photovoltaic generators of the original system were removed because the focus of this work is on the PEV impacts. Fig. 1 shows the single-phase diagram of this radial system, showing only the topology of the MV network.

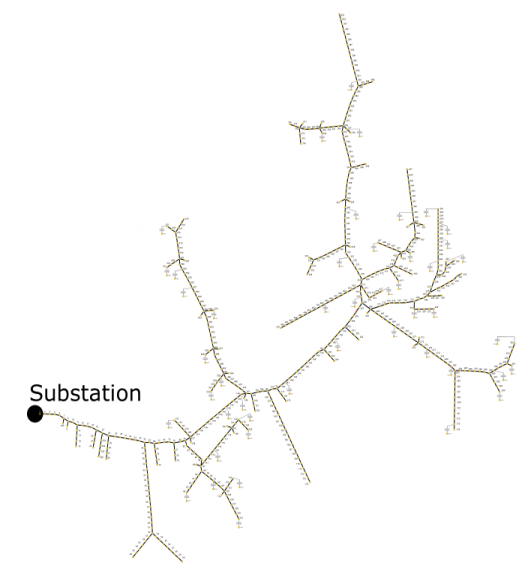

Fig. 1. Single-phase diagram of MV network

The LV networks are three-phase four-wire circuits with multiple single-phase, two-phase and three-phase customers, totaling 1,659 units, and each one of them was a possible PEV charging location. Fig. 2 shows a schematic diagram of the test system with one generic LV network of the 47 under analysis.

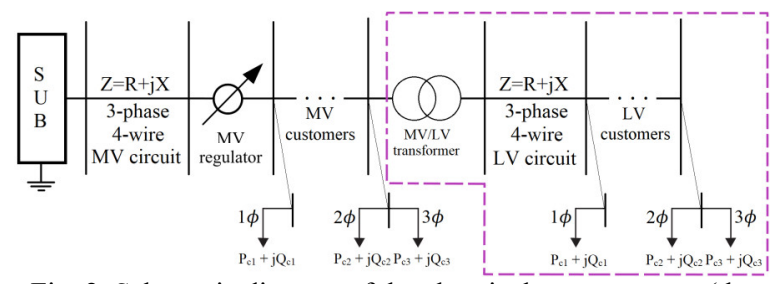

Fig. 2. Schematic diagram of the electrical power system (the purple dotted line represents one generic LV network)

Fig. 3 shows the residential load shape assigned to each LV customer (customer unit - CU) [10], which was modeled as a constant power load. For simplicity, all residential customers are considered with the same load profile.

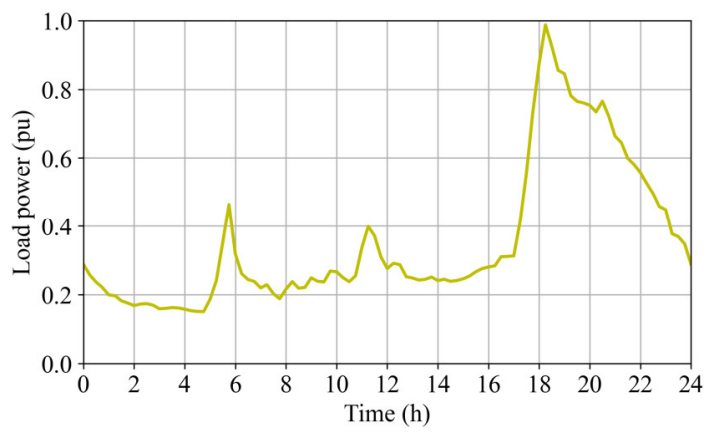

Fig. 3. Customer load shape

The PEVs were modeled as a load with constant power and unitary power factor [11], i.e., consumes energy within it is specified power rating and its stored energy capacity. Based on the characteristics of the Nissan Leaf [12] and the SAE J1772 standard [13], a power of $1.92 \mathrm{~kW}$ was adopted for single-phase charging of level 1 in $127 \mathrm{~V} \mathrm{AC}$ and $6.6 \mathrm{~kW}$ for double-phase charging of level 2 in $220 \mathrm{~V}$ AC. Thus, the main features of the PEV model are present in Table II.

Table II. - Features of the PEV model

\begin{tabular}{|l|c|}
\hline \multicolumn{1}{|c|}{ Name } & Data \\
\hline Battery Capacity & $40 \mathrm{kWh}$ \\
\hline Battery Useable & $36 \mathrm{kWh}$ \\
\hline Electric Range & $240 \mathrm{~km}$ \\
\hline Efficiency & $0.164 \mathrm{kWh} / \mathrm{km}$ \\
\hline Charge Power level 1 & $1.92 \mathrm{~kW}$ \\
\hline Charge Power level 2 & $6.6 \mathrm{~kW}$ \\
\hline
\end{tabular}

In this paper, 127,020 PEV charging profiles were used for level 1 and another 127,020 for level 2 from data provided by [4]. The state of charge (SoC) of the PEV battery, charging duration, and the charging start time are included in the profile developed. Thus, Fig. 4 shows an example of level 1 and level 2 charging profiles. Moreover, uncoordinated PEV residential charging was adopted in this paper. 


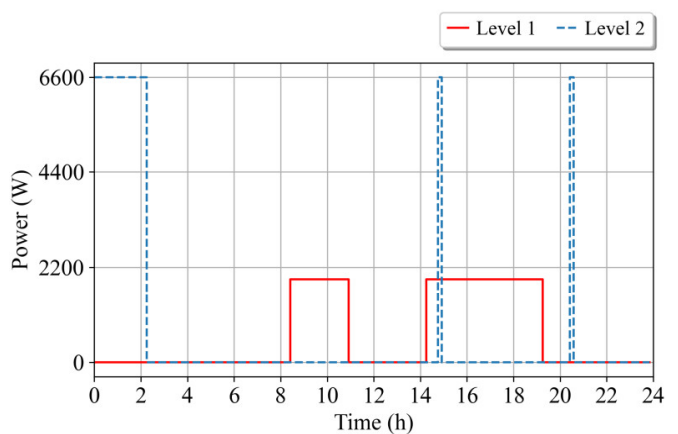

Fig. 4. Example of a level 1 and 2 of the PEV charging profile

\section{Methodology}

The following scenarios are considered:

1) Base scenario: there was no PEV allocated in the DS;

2) $P_{l} 5 \%$ scenario: there were $5 \%$ of $C U s$ with an allocated $P E V$;

3) $P_{l} 15 \%$ scenario: there were $15 \%$ of $C U s$ with an allocated $P E V$;

4) $P_{l} 35 \%$ scenario: there were $35 \%$ of $C U S$ with an allocated $P E V$.

$P_{l}$ means here the percentage resulting from the ratio of the number of customers who have a PEV $\left(C_{P E V}\right)$ to the total number of customers $\left(C_{\text {total }}\right)$ in the entire system, as in (1).

$$
P_{l} \%=\frac{C_{P E V}}{C_{\text {total }}} \cdot 100
$$

Each scenario is simulated for a 24-hour time series. The $\mathrm{PEV}$ is allocated randomly in a CU. Based on the characteristics of the CU (single-phase, two-phase, or three-phase) it was randomly selected a profile of level 1 or 2, where there were 127,020 options for each one under a uniform distribution. Furthermore, the possibility that a $\mathrm{CU}$ has more than one PEV is not considered in this work. After that, OpenDSS performed the power flow through a control code developed in the Python programming language and the results are collected with a 10-min resolution, according to the period required by the Brazilian regulatory agency [14]. In the end, it was carried out the deterministic analysis for each scenario. For example, for the $P_{l} 35 \%$ scenario, more than a third of system CUs had at least one PEV connected and the daily data (144 measurements) for each technical impact under analysis are collected.

\section{Results and Discussion}

This section presents the impacts of PEVs charging on voltage unbalance, power losses and voltage violations in the presence of voltage regulation at the MV network and after the installation of LVR devices.

\section{A. Voltage Unbalance and Technical Losses before LVR}

According to module 8 of the Brazilian Distribution System Procedures (PRODIST) [14], the percentage voltage unbalance factor $(\% V U F)$, or the true definition (TD), is defined as the ratio of the negative sequence voltage $\left(V_{-}\right)$to the positive sequence voltage $\left(V_{+}\right)$, as in (2).

$$
\% V U F=\frac{V_{-}}{V_{+}} \cdot 100
$$

The limits established by regulation for this indicator in Brazil are 2\% for MV networks and 3\% for LV networks. No violation of the VUF limit was recorded in the studied scenarios for the MV network, remaining at values much lower than the limit. On the other hand, Table III shows the maximum $\% V U F\left(\% V U F_{\max }\right)$ obtained for all scenarios in all LV networks.

Table III. - \%VUF $F_{\max }$ for all scenarios at LV networks before LVR

\begin{tabular}{|l|c|}
\hline \multicolumn{1}{|c|}{ Scenarios } & \%VU $\boldsymbol{F}_{\max }(\mathbf{\%})$ \\
\hline Base scenario & 2.27 \\
\hline$P_{l} 5 \%$ scenario & 5.51 \\
\hline$P_{l} 15 \%$ scenario & 4.68 \\
\hline$P_{l} 35 \%$ scenario & 8.95 \\
\hline
\end{tabular}

The predominance of single-phase loads of the base scenario, about $75.9 \%$, was intensified in the LV networks for other scenarios with the presence of singlephase PEVs, contributing to the increase of the $\% V U F$ as the number of PEVs increased. Although there are no penalties for utilities in Brazil for unbalanced systems, this voltage unbalance increases the power losses. In the base scenario it was registered an active loss of $413.474 \mathrm{kWh}$, with $6.71 \%, 23.17 \%$, and $56.37 \%$ increase of active losses for scenarios $P_{l} 5 \%, P_{l} 15 \%$, and $P_{l} 35 \%$, respectively, in comparison with the base scenario. Therefore, the system active losses grew as the penetration level of PEVs increased.

\section{B. Voltage Violations before LVR}

The voltage limits are also established in Brazil by module 8 of PRODIST [14]. The regulation defines that the maximum limit is $1.05 \mathrm{pu}$, for both $\mathrm{MV}$ and $\mathrm{LV}$ networks, and a minimum limit of 0.93 pu for the MV network and $0.92 \mathrm{pu}$ for the LV network, as shown in Fig. 5. Furthermore, if any $\mathrm{CU}$ experiences precarious voltage range for at least $3 \%$ of the period under analysis (e.g. one day) or critical voltage range for at least $0.5 \%$ of the analyzed period, that $\mathrm{CU}$ is eligible for compulsory compensation by utilities.

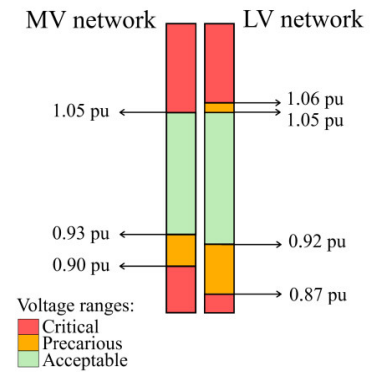

Fig. 5. Acceptable voltage ranges according to PRODIST for MV and LV systems 
Fig. 6 shows the maximum (Vmax) and minimum (Vmin) voltages of the system obtained for all scenarios.

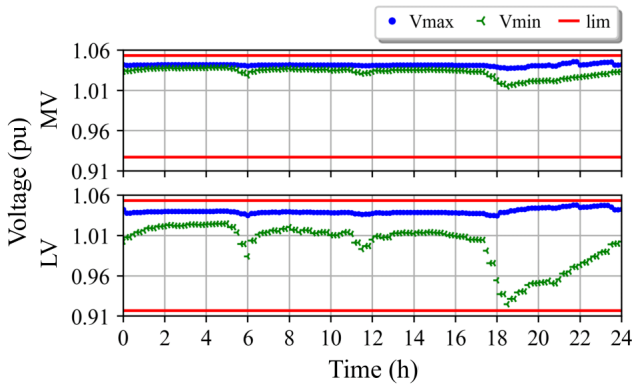

(a)

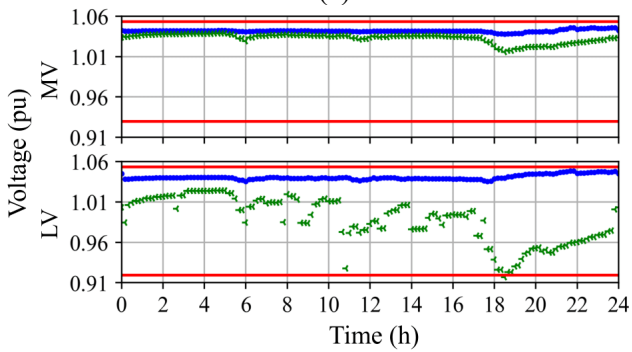

(b)

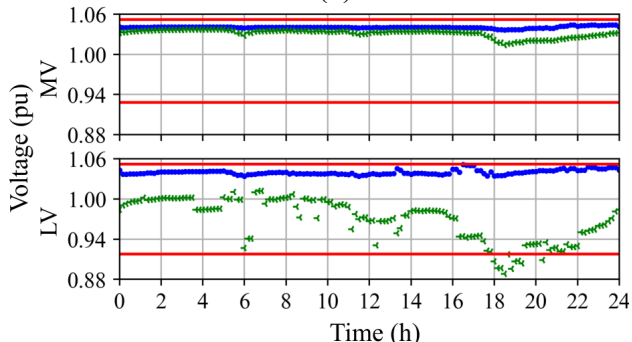

(c)

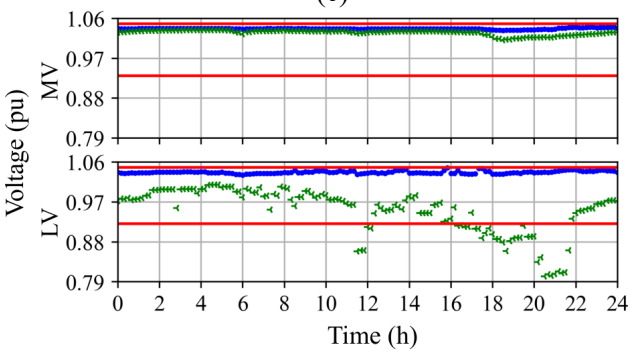

(d)

Fig. 6. Maximum and minimum voltages before LVR devices. a) Base scenario. b) $P_{l} 5 \%$ scenario. c) $P_{l} 15 \%$ scenario. d) $P_{l} 35 \%$ scenario.

The results have shown that there were violations of the minimum voltage limit for all scenarios, except for the base scenario. It is worthwhile to point out that voltage violations occurred even for a low penetration level scenario, e.g. $P_{l} 5 \%$. Furthermore, the voltage drop was very intense during some periods, reaching $0.798 \mathrm{pu}$ in the scenario $P_{l} 35 \%$. Another important result is that the DS witnessed voltage increase in some phases/buses of the LV networks in all scenarios, but with no violation of the maximum voltage limit.

\section{Voltage Regulation at MV Network}

The system under study did not present any automatic voltage regulator in its original version provided by [8].
However, for developing this work, an automatic voltage regulator was inserted into the MV network, located on the feeder's main trunk. Thus, Fig. 7 depicts the tap positions of the MV regulator for the three phases of the system.

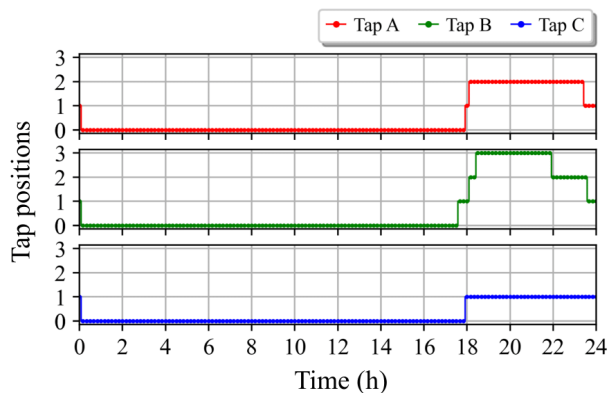

(a)

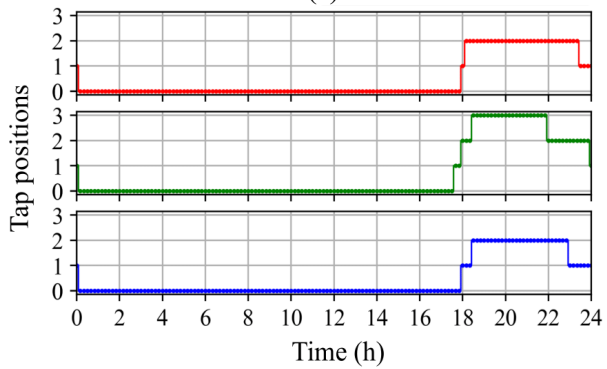

(b)

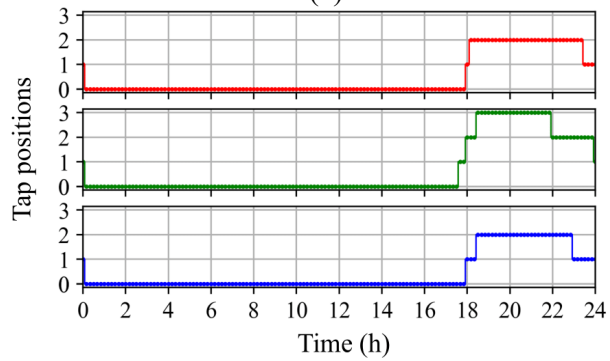

(c)

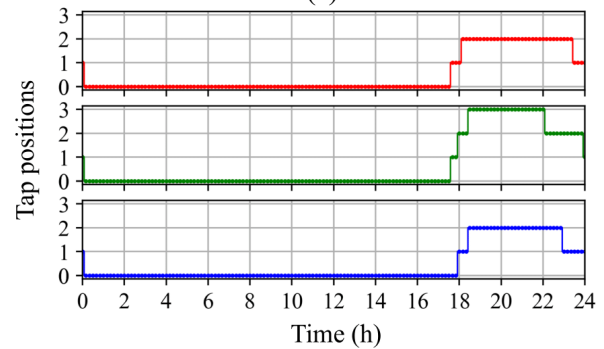

(d)

Fig. 7. Tap positions of the MV regulator. a) Base scenario. b) $P_{l} 5 \%$ scenario. c) $P_{l} 15 \%$ scenario. d) $P_{l} 35 \%$ scenario.

The results showed that there were few tap changes when comparing the PEV penetration scenarios with the base scenario. Moreover, the tap changes were not able to correct the voltage violations in the LV networks, as well presented previously in Subsection B, because the voltages at the MV level were within acceptable limits. Therefore, this finding leads to the implementation of LVR devices at the studied system as a solution to the violations of the steady-state voltage limits in the LV networks, since it was the most restrictive impact on the studied system. Also, the results presented in section D consider the MV regulator and the LVRs devices operating together in the system. 


\section{Voltage Unbalance and Technical Losses after LVR}

The LVR devices were modeled according to the data provided by [15]. It consists of a commercial regulator for each phase of the system, with a regulation range of $\pm 10 \%$, in 8 -steps (nominal voltage is $0.22 \mathrm{kV}$ ). The computational model of the automatic regulator was made available by OpenDSS [9]. Each wye-connected voltage regulator was sized in line with the size of distribution transformers chosen for the implementation of LVR devices (designed to maintain an output voltage at 1.02 pu). Thus, Fig. 8 shows a generic schematic diagram of the LVR implementation.

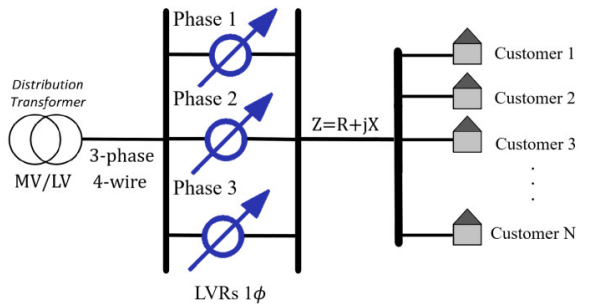

Fig. 8. The generic schematic diagram for LVR implementation

The nine most loaded LV networks out of the 47 present in the studied system are selected for LVRs implementation and the simulations are performed again. Then, the new results are presented in Table IV for $\% V U F_{\max }$.

Table IV. - \%VUF $\max$ for all scenarios at LV networks after LVR

\begin{tabular}{|l|c|}
\hline \multicolumn{1}{|c|}{ Scenarios } & \%VU $\boldsymbol{F}_{\max }$ \\
\hline Base scenario & 1.83 \\
\hline$P_{l} 5 \%$ scenario & 3.37 \\
\hline$P_{l} 15 \%$ scenario & 4.54 \\
\hline$P_{l} 35 \%$ scenario & 6.24 \\
\hline
\end{tabular}

The results show that the \%VUF was improved in comparison with the results presented in Table III, most in part because the LVR devices were single-phase and regulated each phase separated. However, the losses obtained after the implementation of the LVRs are slightly higher than the previous ones, as shown in Table V.

Table V. - Power losses

\begin{tabular}{|l|c|c|}
\hline \multirow{2}{*}{ Scenarios } & \multicolumn{2}{|c|}{ Active power losses (kWh) } \\
\cline { 2 - 3 } & Before LVR & After LVR \\
\hline Base scenario & 413.474 & 418.203 \\
\hline$P_{l} 5 \%$ scenario & 441.233 & 448.298 \\
\hline$P_{l} 15 \%$ scenario & 509.291 & 511.782 \\
\hline$P_{l} 35 \%$ scenario & 646.559 & 637.317 \\
\hline
\end{tabular}

This increase in the losses among the scenarios was expected before LVR implementation, since each PEV represents an additional load to the system. Thus, the higher the $P_{l}$, the greater the losses. After the LVR devices, allocation in the LV networks losses increased more usually due to the insertion of new equipment in the networks, which represents an additional impedance to the system. However, an exception occurs in $P_{l} 35 \%$ scenario, where the losses measurement indicates that there was a reduction compared to the system before LVR. This occurred due to single-phase PEVs charging. The PEV charging allocation could soften the $\% V U F$ of the system and reduce the grid loss since the PEVs were better distributed. Nevertheless, this had nothing to do with the implementation of LVRs, which gently increase losses, as stated earlier. Although this outcome not following the trend of the other scenarios analyzed, the increase of technical losses could be a disadvantage of this method and need better evaluation. It stands out there is no technical restriction limiting the increase in electrical losses; this is an economic issue for utilities.

\section{E. Voltage Violations after LVR}

The maximum (Vmax) and minimum (Vmin) voltages obtained for all scenarios after the implementation of all nine LVR devices in the system under analysis is depicted in Fig. 9.

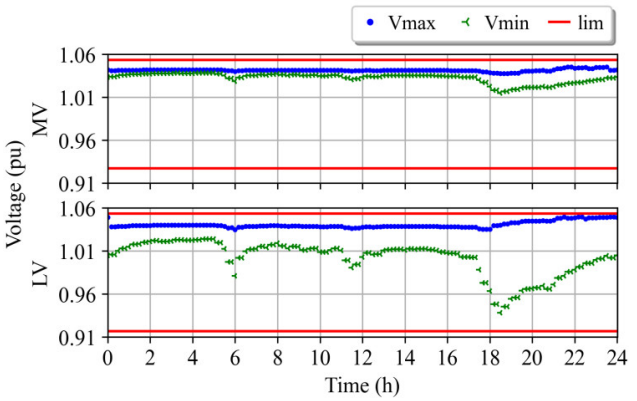

(a)

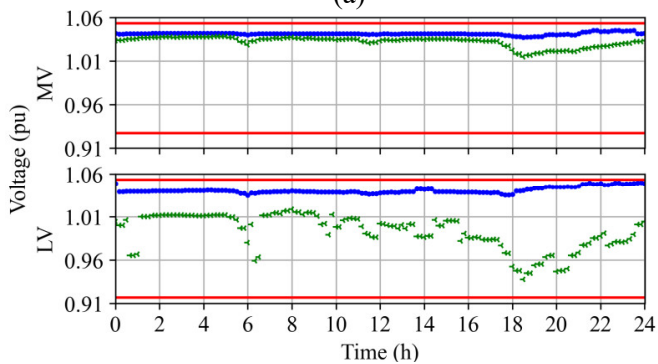

(b)

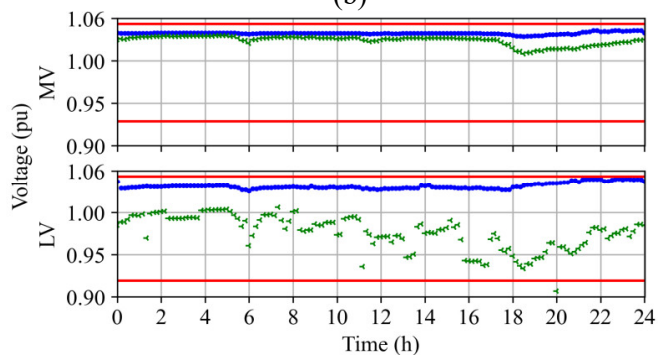

(c)

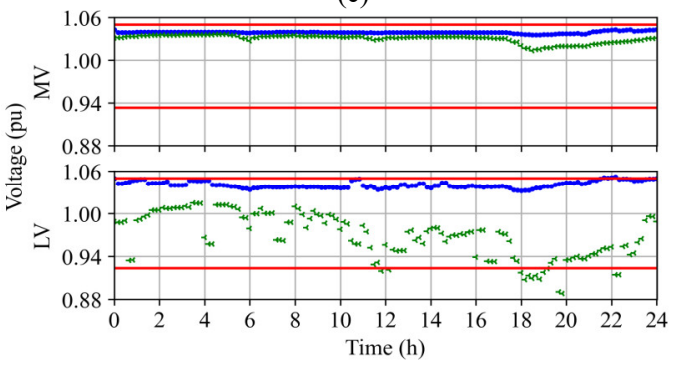

(d)

Fig. 9. Maximum and minimum voltages after LVR devices. a) Base scenario. b) $P_{l} 5 \%$ scenario. c) $P_{l} 15 \%$ scenario. d) $P_{l} 35 \%$ scenario. 
Voltage magnitude in the LV networks improved after the implementation of the LVR devices compared with the results shown in Fig. 6 (before LVR devices). In this case, the worst voltage drop corresponding to a magnitude of $0.89 \mathrm{pu}$, for the $P_{l} 35 \%$ scenario, against $0.798 \mathrm{pu}$ verified for the same scenario in Section IV.B. Thus, with the LVR devices, utilities could avoid penalties for violation of voltage for a $P_{l}$ of up to $15 \%$, reducing company costs and improving the service quality. In addition, a simulation was carried out varying the short circuit level to $100 \mathrm{MVA}$ and 1000 MVA (the original value previously used to compose the results section of this paper was 500 MVA). For $P_{l} 35 \%$ scenario, e.g., considering the short circuit level at 100 MVA, 10 different periods of a day were observed with voltage violations (minimum $0.86 \mathrm{pu}$ ). For 1000 MVA, only 3 periods with voltage violations were observed (minimum $0.89 \mathrm{pu}$ ). While for $500 \mathrm{MVA}$, as shown in Fig. 9d, 11 periods were registered (minimum $0.88 \mathrm{pu})$. As the level of the system's short circuit increases, the number of violations drops. This behavior was also seen in the other scenarios.

\section{Conclusion}

This paper presented an investigation on using LVR to minimize the impacts of charging PEVs in DS. The voltage drop was one of the most serious impacts resulting from the connection of PEVs to the electrical grid. Furthermore, the uncontrolled charging of PEVs can result in both a decrease and increase of the $\% V U F$ among the scenarios, leading to severe violations. Moreover, the more unbalanced the system becomes, the higher the active losses, which also grow due to the PEV load increase.

It is important to highlight that the voltages in the LV networks exceeded the limits, but the same did not occur for the MV network. For this reason, the automatic voltage regulator has not mitigated all the violations in the LV networks. Therefore, nine LVR devices were implemented in the system under analysis, and the results have shown that the LV networks where the LVRs were allocated experienced an improvement of the voltage profile of the system as a whole. The $\% V U F$ was reduced, since the LVRs were single-phase and could operate individually. Also, it is worthwhile to point out that the voltage violations were significantly reduced too, but the LVR devices did not prevent them to occur. The LVRs prevented violations in the critical voltage range for all scenarios, which is the worst-case scenario, avoiding compulsory compensation by utilities for this range.

Therefore, in cases where controlled PEV charging strategies may not be available to PEVs owners, thus applying grid-based solutions, such as LVR, could be an interesting and feasible approach for utilities. Nevertheless, deterministic analysis, as used in this work, could be a limited approach and stochastic simulations are proposed to be employed as a future improvement for more comprehensive analyses.

\section{Acknowledgments}

This work was supported in part by the Coordenação de Aperfeiçoamento de Pessoal de Nível Superior - Brasil (CAPES) - Finance Code 001 and by the Conselho Nacional de Desenvolvimento Científico e Tecnológico (CNPq), under Grants 134232/2019-4 and 421310/20189.

\section{References}

[1] WEF, "Electric Vehicles for Smarter Cities: The Future of Energy and Mobility," Geneva, 2018.

[2] IEA, "Global EV Outlook 2021," Paris, 2021.

[3] O. Polat, O. H. Eyüboglu and O. Gül, "Monte carlo simulation of electric vehicle loads respect to return home from work and impacts to the low voltage side of distribution network", Electrical Engineering, vol. 103, pp. 439-445, 2021.

[4] M. Muratori, "Impact of uncoordinated plug-in electric vehicle charging on residential power demand," Nature Energy, pp. 193-201, 2018.

[5] T. Barbosa, J. Andrade, R. Torquato, W. Freitas and F. C. L. Trindade, "Use of EV hosting capacity for management of low-voltage distribution systems," IET Generation, Transmission Distribution, vol. 14, pp. 2620-2629, 2020.

[6] N. Efkarpidis, T. D. Rybel e J. Driesen, “Optimal Placement and Sizing of Active In-Line Voltage Regulators in Flemish LV Distribution Grids," IEEE Transactions on Industry Applications, vol. 52, pp. 4577 4584, 2016.

[7] H. Kikusato, J. Yoshinaga, Y. Fujimoto, Y. Hayashi, S. Kusagawa e N. Motegi, "Deployment of low-voltage regulator considering existing voltage control in mediumvoltage distribution systems," Journal of International Council on Electrical Engineering, vol. 6, pp. 252-260, 2016.

[8] L. C. Brolin, F. B. B. Rolim e F. C. L. Trindade, "Fast screening approach to estimate the hosting capacity of rooftop PV generators in electric power distribution systems," International Transactions on Electrical Energy Systems, vol. 29, p. e2740, 2019.

[9] EPRI, "Open Distribution System Simulator (OpenDSS)," 2021.

[10] EPRI, "EPRI Test Circuits,” 2021. Available: http://svn.code.sf.net/p/electricdss/code/trunk/Distrib/

[11] J. Su, T. T. Lie e R. Zamora, "Integration of Electric Vehicles in Distribution Network Considering Dynamic Power Imbalance Issue," IEEE Transactions on Industry Applications, vol. 56, pp. 5913-5923, 2020.

[12] EV Database, Nissan Leaf, 2021. Available: https://evdatabase.org/car/1106/Nissan-Leaf

[13] S. A. E. International, "SAE J1772: Electric Vehicle and Plug in Hybrid Electric Vehicle Conductive Charge Coupler," PA, 2017.

[14] ANEEL, "Module 8," Brazilian Distribution System Procedures (PRODIST), Brazil, 2021 (in portuguese).

[15] A. E. GmbH, Low voltage regulation system LVRSys ${ }^{\mathrm{TM}}$ Data Sheet, Nuremberg, Germany: A. Eberle GmbH, 2020. 